\title{
OPTIONAL FRONT END MODELS FOR THE NEUTRINO FACTORY STUDY II
}

\author{
Yasuo Fukui* UCLA \\ MS 26, SLAC, Stanford Univ., P.O.Box 4349, Stanford, CA 94309, USA
}

\begin{abstract}
Options of the phase rotation and the ionization cooling channels was studied in the Neutrino Factory Study II where a muon storage ring is assumed to be built at BNL. Quality of the high intensity muon beam was studied as function of the modified parameters of the front end channels which consists of the pion capture system, nondistorting phase rotation system, and the ionization cooling channel. The purpose of the study is to find the possible start-up options of the system and their performance while the part of the channel elements are available.
\end{abstract}

\section{INTRODUCTION}

Historically, the pion capture and the phase rotation channel has been designed by using RF cavities with frequency ranges of $20-100 \mathrm{MHz}$ in order to produce a single muon bunch of $\mu^{+}$or $\mu^{-}$as the beam source of a $\mu^{+} \mu^{-}$ collider. [1, 2] A complete beam cooling model for the $\mu^{+} \mu^{-}$collider has yet to be build. For the $\nu$ factory design, the goal is to let the muons decay in a straight section of a storage ring, and the requirement on the 6 dimensional normalized emittance of the muon beam is smaller than that for the $\mu^{+} \mu^{-}$collider by a factor of $10^{(4-5)}$. The length of the final muon beam can be as long as the circumference of the storage ring. $[4,5]$ In this study, we studied the performance degradation of the $\nu$ Factory Feasibility Study II front end channel by removing the first Induction Linac Channel or the second Induction Linac Channel and the transverse phase space cooling liq. Hydrogen absorber. The non-distorting phase rotation system is used in the $\nu$ Factory Feasibility Study II, where the density of muons as a function of ct, $\frac{d N}{d(c t)}$, is kept uniform and $\frac{d N}{d p_{z}}$ is independent of ct. by using the two stages of the induction linac channel and a liq. $H_{2}$ absorber and a drift space in between them. [8]

\section{PERFORMANCE STUDY}

\subsection{Performance of the Baseline Model}

Schematic diagram of the channel components in $\mathrm{r}-\mathrm{z}$ plane is sketched in Figure 1. The top is the model of the $\nu$ Factory Feasibility Study II. The second from top is the front end channel without the first induction linac channel and the liq. $\mathrm{H}_{2}$ absorber. The third from top is the one without the liq. $\mathrm{H}_{2}$ absorber and the second induction linac channel. A model for the $\nu$ Factory Feasibility Study I is sketched at the bottom as a reference. The total length of

\footnotetext{
*fukui@slac.stanford.edu
}

the $\nu$ Factory Feasibility Study $\mathrm{I}$ is almost half of that in the $\nu$ Factory Feasibility Study II.
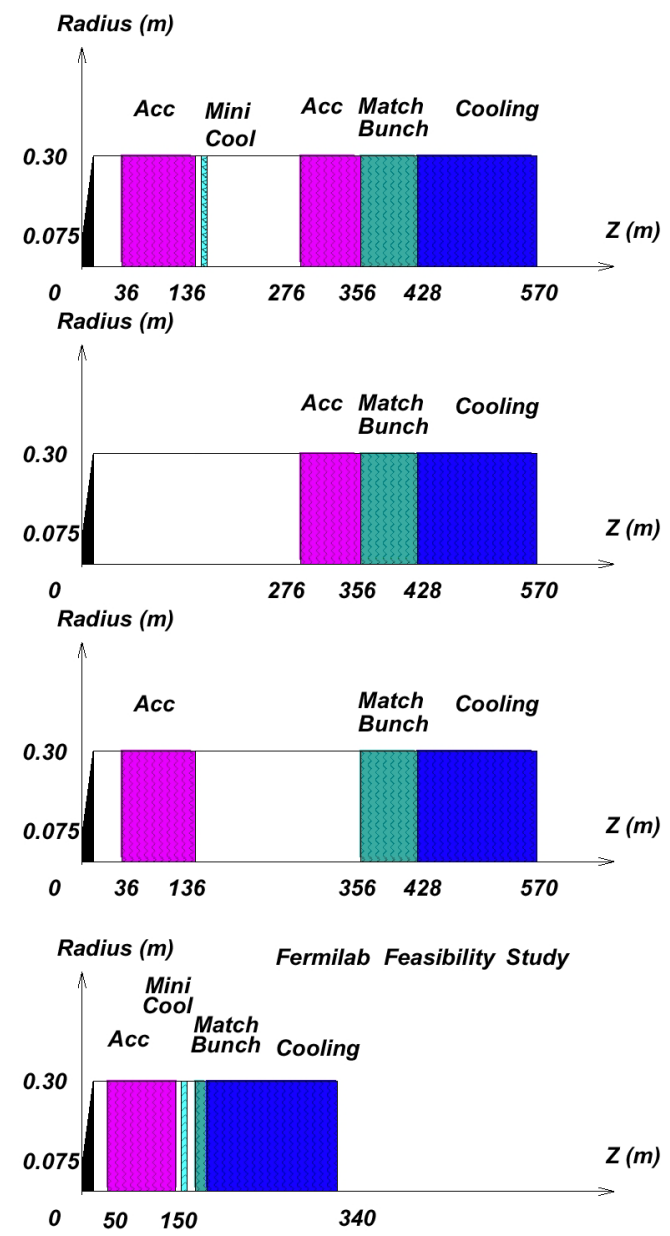

Figure 1: Schematic Front End Channels of Plan A(Top), Plan C(Middle), Study 1(Bottom)

Kinetic Energy vs. z, and Relative Arrival Time vs. z are plotted in Figure 2 with 3 sample events in the Study II model. The feature of the non-distorting phase rotation system, where the relative arrival time of the muons at the end of the cooling channel is almost proportional to the initial kinetic energy of muons at the $\mathrm{z}$ location close to the target. In the first induction linac channel, lower kinetic energy muons were boosted, and the kinetic energy of the all muons was reduced through a liq. $H_{2}$ mini-cooler, which increases the deviation of the velocity $(\beta)$, which make the following drift space more effective to expand the muon bunch length. 


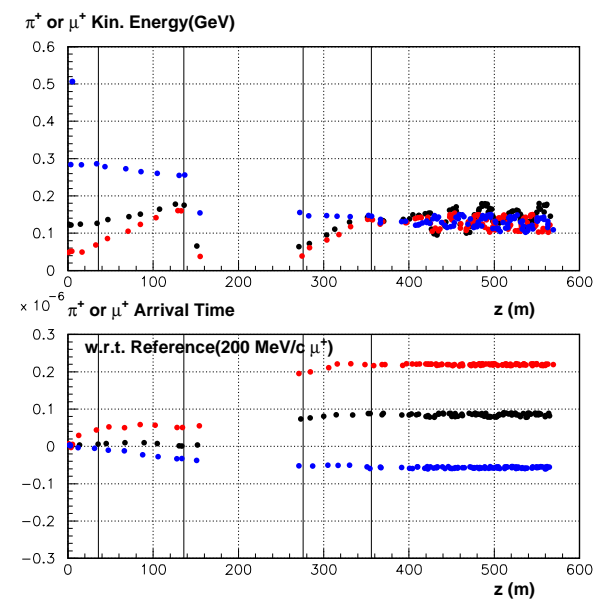

Figure 2: Kinetic Energy vs. z, and Relative Arrival Time vs. $\mathrm{z}$ with 3 example events in Plan A model

\subsection{Performance of the Model with a Partial In- duction Linac Channel}

Figure 3 shows the longitudinal $\mu^{+}$phase space $\left(p_{z}\right.$ vs. t) at the entrance and exit of the 1st and 2nd Induction Linac channels in the Study II model(top), Use only 2nd Ind. Linac(middle) Use only 1std Ind. Linac(bottom).

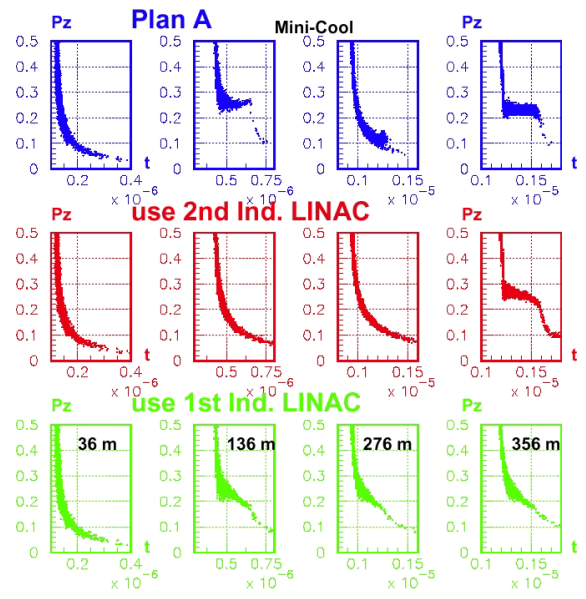

Figure 3: Long. $\mu^{+}$phase space at the entrance/exit of the 1st, 2nd Induction Linac in Plan A(TOP), Use only 2nd Ind. Linac(Middle) Use only 1std Ind. Linac(Bottom)

$\mu^{+} \mathrm{Pz}$ and $\mathrm{t}$ distribution at the exit of the 2nd Induction Linac in the study II model, in the case of using only 2 nd Induction Linac channel, and in the case of using only 1st Induction Linac channel are shown in Figure 4.

$\left(\mu^{+}\right.$or $\left.\pi^{+}\right)$/primary proton ratio as a function of $\mathrm{z}$ are shown in Figure 5 from the target to the end of the cooling
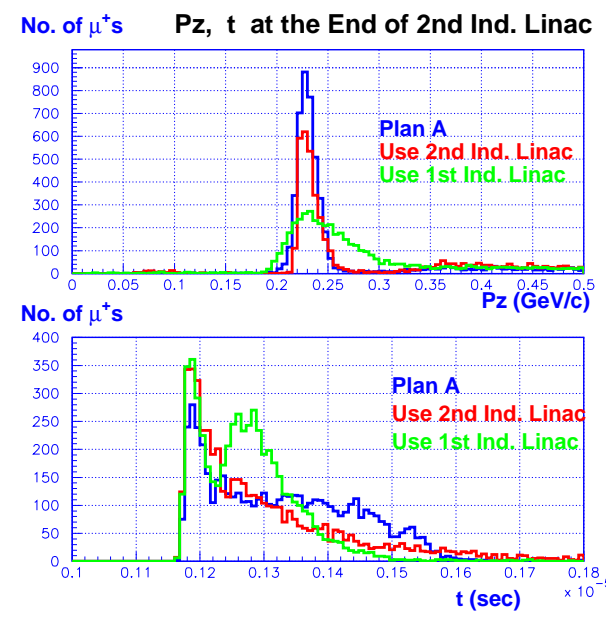

Figure 4: $\mu^{+} \mathrm{Pz}$ and $\mathrm{t}$ distribution at the exit of the 2nd Induction Linac in Plan A(blue), Use only 2nd Ind. Linac(red) Use only 1st Ind. Linac(green)

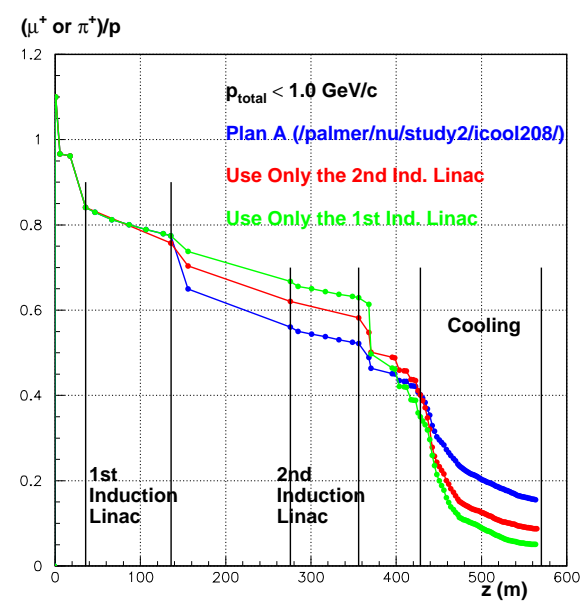

Figure 5: $\left(\mu^{+}\right.$or $\left.\pi^{+}\right) /$primary proton ratio as a function of Z

channel in the study II model, in the case of using only 2nd Induction Linac channel, and in the case of using only 1st Induction Linac channel.

\section{CONCLUSION}

Removing the First or the Second Induction Linac Channel and the Mini-Cooling is shown to degrade the phase rotation and cooling performance in this study.

The ultimate cost-saving front end model can be a set of a phase rotation ring, (FFAG, for example) a cooling ring, an emittance exchange(bunch stacking)/cooling ring. A figure of merit will be a total 1-turn channel length. 


\section{ACKNOWLEDGMENTS}

The authors wish to thank A Sessler, M. Zisman, J. Wurtele, C. Kim, E. Kim, G. Penn, N. Holtkamp, P. Lebrun, D. Neuffer, V. Balbekov, N. Mokhov, A. Lombardi, and R. Scrivens for the useful discussions, R. B. Palmer and $\mathrm{H}$. Kirk for the general phase rotation idea, R. Fernow and J. Gallardo, and , D. Cline for their help and encouragement.

\section{REFERENCES}

[1] R. B. Palmer et al., " $\mu^{+} \mu^{-}$Collider: a Feasibility Study"

Snowmass96 workshop proceedings, BNL-52503, Fermilab-Conf.-96.092, LBNL-38946

[2] C. M. Ankenbrandt et al.,

"Status of muon collider research and development and future plans"

Phys. Rev. ST Accel. Beams 2, 081001

[3] R. B. Palmer et. al.,Neutrino Factory Draft Parameters Muon Collider Note 46, September 3, 1999

[4] T. Anderson et al.,

"A Feasibility Study of a Neutrino Source Based on a Muon Storage Ring"

Fermilab report, March, 2000

[5] B. Palmer et al.,

"A BNL Feasibility Study II Report"

to be submitted, April, 2001

[6] N. V. Mokhov

"The MARS Code System User's Guide, Version 13(98)"

Fermilab-FN-628, 1998

[7] R. Fernow

"A Simulation Code for Ionization Cooling of Muon Beams"

submitted to PAC99

[8] R. Palmer

"Non-distorting phase rotation"

Muon Collider Note No. 114, April 2000 